\title{
An Approximate Analytical Solution of Sloshing Frequencies for a Liquid in Various Shape Aqueducts
}

\author{
Yuchun Li ${ }^{1}$ and Zhuang Wang ${ }^{2}$ \\ ${ }^{1}$ Department of Hydraulic Engineering, Tongji University, 1239 Siping Road, Shanghai 200092, China \\ ${ }^{2}$ Research Institute of Structural Engineering and Disaster Reduction, Tongji University, 1239 Siping Road, Shanghai 200092, China \\ Correspondence should be addressed to Yuchun Li; ycl2000@tongji.edu.cn
}

Received 26 February 2013; Accepted 3 July 2013; Published 13 February 2014

Academic Editor: Ahmet S. Yigit

Copyright (c) $2014 \mathrm{Y}$. Li and Z. Wang. This is an open access article distributed under the Creative Commons Attribution License, which permits unrestricted use, distribution, and reproduction in any medium, provided the original work is properly cited.

\begin{abstract}
An approximate analytical solution of sloshing frequencies for a liquid in the various shape aqueducts is formulated by using the Ritz method. The present approximate method is, respectively, applied to find the sloshing frequencies of the liquid in rectangular, trapezoid, oval, circular, U-shaped tanks (aqueducts), and various shape tuned liquid dampers (TLD). The first three antisymmetric and symmetric frequencies by the present approach are within 5\% accuracy compared to the other analytical, numerical, and experimental values. The approximate solutions of this paper for the various shape aqueducts are acceptable to the engineering applications.
\end{abstract}

\section{Introduction}

Sloshing is a common physical phenomenon which occurs in moving tanks with contained liquid masses, such as liquid cargo carriers, rockets, aircrafts, and the seismically excited storage tanks, dams, reactors, and nuclear vessels. The sloshing frequencies of contained liquid are essential in the analysis and design of the liquid tanks and the associated structures.

The modal analysis of the linearized free liquid surface in a rigid container has been widely studied by many scholars [1-4]. The various methods of modal analysis were well documented in the books by Ibrahim [3] and Faltinsen and Timokha [4]. These approaches are, respectively, variational formulation (Lawrence et al. [5]), integral equation (Budiansky [6]), conformal mapping (Fox and Kuttler [7]), bipolar coordinate transformation (McIver [8]), and so forth. The analytical and approximate methods to estimating the sloshing frequencies were developed by Evans [9] and Evans and Linton [10]. The numerical methods [11] in common use are the finite difference, finite element, and boundary element. The finite element may be the most commonly used approach in the sloshing analyses. On the basis of the small displacement assumption, the irrotational fluid can be modeled by a degenerate solid finite element $[12,13]$ with no shear resistance.
This displacement-based finite element formulation, which had been introduced into the commercial ANSYS Code [14], was usually used for simulating the sloshing problems $[15,16]$ in certain engineering fields.

The sloshing frequencies of a liquid in an arbitrarysection aqueduct can be accurately extracted by the above mentioned methods. However, it is not easy work. For many engineering problems, the engineers only need the approximate previous order frequencies. The purpose of this paper is to provide a simple and quick method to evaluate the sloshing frequencies of a liquid in the various shape aqueducts.

\section{Basic Equations of Two-Dimensional Free Sloshing}

An arbitrary-section aqueduct is shown in Figure 1, where the 2-D Cartesian coordinates $o x z$ are fixed to the aqueduct and the symbols $\Omega, \partial S_{f}$, and $\partial S_{w}$, respectively, denote the static liquid region, the free liquid surface, and the wetted boundary. The $x$ axis is in the plane of free surface and $y$ axis points vertically upwards through the midpoint of the free surface. The width of free surface is $2 a$. The aqueduct is presumed to be rigid. 


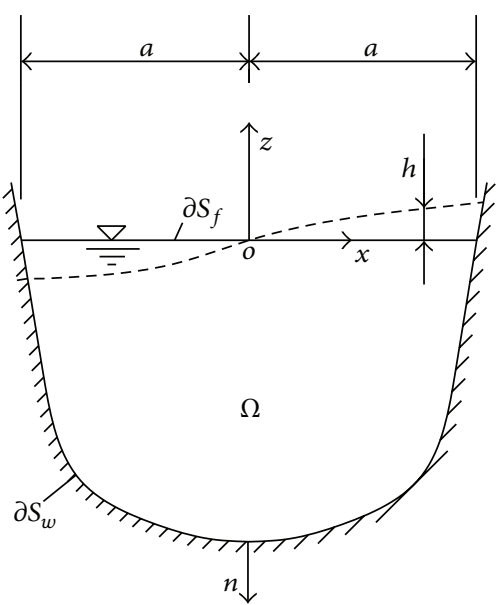

Figure 1: An arbitrary-section aqueduct.

On the basis of the linear potential theory [1-4], the fluid in absence of surface tension on the free surface can be assumed to be inviscid, incompressible, irrotational, and of small displacement; the two-dimensional free sloshing of a liquid inside a motionless aqueduct can be described by the following boundary value problem:

$$
\begin{gathered}
\nabla^{2} \Phi(x, z, t)=0 \\
\left.\frac{\partial \Phi(x, z, t)}{\partial n}\right|_{\partial S_{w}}=0 \\
{\left.\left[\frac{\partial \Phi(x, z, t)}{\partial z}-\frac{\partial h(x, z, t)}{\partial t}\right]\right|_{\partial S_{f}}=0} \\
{\left.\left[\frac{\partial \Phi(x, z, t)}{\partial t}+g h(x, z, t)\right]\right|_{\partial S_{f}}=0}
\end{gathered}
$$

where $\Phi(x, z, t)$ is the function of velocity potential, $h(x, z, t)$ is the wave-height function on the free surface, $t$ is the time, $g$ is the acceleration of gravity, $n$ is the normal drawn outwardly on the wetted boundary (see Figure 1), and $\partial / \partial n$ denotes differentiation in the direction of the normal.

\section{Approximate Analytical Solution}

In order to find the solutions of (1)-(4), we may proceed directly by assuming trial solutions:

$$
\begin{gathered}
\Phi(x, z, t)=i \omega \varphi(x, z) \exp (i \omega t), \\
h(x, z, t)=f(x, z) \exp (i \omega t),
\end{gathered}
$$

where $i=\sqrt{-1}, \omega$ is the sloshing (circular) frequency and $\varphi(x, z)$ and $f(x, z)$ are, respectively, the amplitude functions of $\Phi(x, z, t)$ and $h(x, z, t)$. Substituting (5) into (1)-(4) and merging (3) and (4) into a single equation, we arrive at the following eigenvalue problem:

$$
\begin{gathered}
\nabla^{2} \varphi(x, z)=0, \quad(x, z) \in \Omega, \\
\left.\frac{\partial \varphi(x, z)}{\partial n}\right|_{\partial S_{\omega}}=0 \\
{\left.\left[\frac{\partial \varphi(x, z)}{\partial z}-\frac{\omega^{2}}{g} \varphi(x, z)\right]\right|_{\partial S_{f}}=0 .}
\end{gathered}
$$

The sloshing frequency can also be expressed as $[1,3]$

$$
\omega^{2}=\frac{g \iint_{\Omega}\left[(\partial \varphi(x, z) / \partial x)^{2}+(\partial \varphi(x, z) / \partial z)^{2}\right] d x d z}{\int_{\partial S_{f}} \varphi^{2}(x, z) d s} .
$$

From (8) and (9), we further obtain

$$
\begin{gathered}
\omega^{2}=g \frac{\left.(\partial \varphi(x, z) / \partial z)\right|_{z=0}}{\left.\varphi(x, z)\right|_{z=0}}, \\
\frac{\iint_{\Omega}\left[(\partial \varphi(x, z) / \partial x)^{2}+(\partial \varphi(x, z) / \partial z)^{2}\right] d x d z}{\int_{-a}^{a} \varphi^{2}(x, z) d x} \\
=\frac{\left.(\partial \varphi(x, z) / \partial z)\right|_{z=0}}{\left.\varphi(x, z)\right|_{z=0}} .
\end{gathered}
$$

For the $j$ th frequency $\omega_{j}(j=1,2,3, \ldots)$ and mode function $\varphi_{j}(x, z)(j=1,2,3, \ldots)$, they both satisfy (10) and (11). It can be seen from (10) that the sloshing frequency $\omega_{j}$ exclusively depends on the mode function $\varphi_{j}(x, z)$. In order to obtain an approximate frequency $\omega_{j}$, the function $\varphi_{j}(x, z)$ can be approximately assumed to be a product of two individual functions $\xi_{j}(x)$ and $\eta_{j}(z)$ of the coordinates; that is, $\varphi_{j}(x, z)=$ $\xi_{j}(x) \cdot \eta_{j}(z)$. In the physical sense, the function $\xi_{j}(x)$ presents the $j$ th mode shape of free liquid surface. On the basis of the Ritz method, $\xi_{j}(x)$ can be approximately expressed as a known trigonometric function $\sin \alpha_{j} x$ for the antisymmetric mode or $\cos \beta_{j} x$ for the symmetric mode, in which $\alpha_{j}, \beta_{j}$ are the known constants. Inserting $\varphi_{j}(x) \approx \sin \alpha_{j} x \cdot \eta_{j}(z)$ or $\varphi_{j}(x, z) \approx \cos \beta_{j} x \cdot \eta_{j}(z)$ into (6), we can find the solution $\eta_{j}(z)=A_{j}\left(\cosh \alpha_{j} z+B_{j} \sinh \alpha_{j} z\right)$ or $\eta_{j}(z)=$ $C_{j}\left(\cosh \beta_{j} z+D_{j} \sinh \beta_{j} z\right)$, in which $A_{j}, B_{j}, C_{j}$, and $D_{j}$ $(j=1,2,3, \ldots)$ are the undetermined coefficients. Because the coefficients $A_{j}$ and $C_{j}$ have no effect on the final outcomes of the frequencies, they are ignored here. Finally the mode function $\varphi_{j}(x, z)$, corresponding to the $j$ th antisymmetric and symmetric modes, can be proximately presented as

$$
\begin{aligned}
& \varphi_{j}(x, z) \\
& \approx\left\{\begin{array}{c}
\sin \alpha_{j} x \cdot\left(\cosh \alpha_{j} z+B_{j} \sinh \alpha_{j} z\right) \\
\text { antisymmetric mode } \\
\cos \beta_{j} x \cdot\left(\cosh \beta_{j} z+D_{j} \sinh \beta_{j} z\right) \\
\text { symmetric mode }
\end{array} \quad(j=1,2,3, \ldots),\right.
\end{aligned}
$$




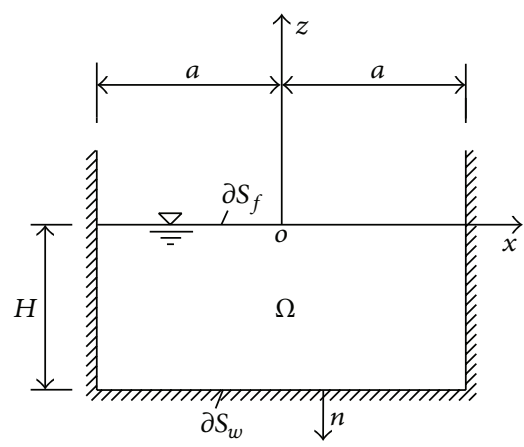

FiguRe 2: A rectangular tank.

where

$$
\alpha_{j}=\frac{(2 j-1) \pi}{2 a}, \quad \beta_{j}=\frac{j \pi}{a} .
$$

Thus, substitution of (12) into (10) yields the antisymmetric and symmetric sloshing frequencies

$$
\omega_{j}^{2} \approx\left\{\begin{array}{ll}
B_{j} \alpha_{j} g & \text { antisymmetric } \\
D_{j} \beta_{j} g & \text { symmetric }
\end{array} \quad(j=1,2,3, \ldots) .\right.
$$

It is noted that the function $\varphi_{j}(x, z)$ has only one unknown coefficient $B_{j}$ (or $D_{j}$ ), which can be uniquely determined by (11). Substituting (12) into (11), we, respectively, acquire the two quadratic equations with the unknowns $B_{j}$ and $D_{j}$ :

$$
\begin{aligned}
& a_{A j} B_{j}^{2}+b_{A j} B_{j}+c_{A j}=0, \quad(j=1,2,3, \ldots), \\
& a_{S j} D_{j}^{2}+b_{S j} D_{j}+c_{S j}=0, \quad(j=1,2,3, \ldots),
\end{aligned}
$$

where

$$
\begin{gathered}
a_{A j}=\iint_{\Omega}\left(\sin ^{2} \alpha_{j} x+\sinh ^{2} \alpha_{j} z\right) d x d z, \\
b_{A j}=2 \iint_{\Omega} \sinh \alpha_{j} z \cdot \cosh \alpha_{j} z \cdot d x d z-\frac{a}{\alpha_{j}}, \\
c_{A j}=\iint_{\Omega}\left(\cos ^{2} \alpha_{j} x+\sinh ^{2} \alpha_{j} z\right) d x d z, \\
\left.a_{S j}=\iint_{\Omega}\left(\cos ^{2} \beta_{j} x+\sinh ^{2} \beta_{j} z\right) d x d z, 2, \ldots\right), \\
b_{S j}=2 \iint_{\Omega} \sinh \beta_{j} z \cdot \cosh \beta_{j} z \cdot d x d z-\frac{a}{\beta_{j}}, \\
c_{S j}=\iint_{\Omega}\left(\sin ^{2} \beta_{j} x+\sinh ^{2} \beta_{j} z\right) d x d z, \\
(j=1,2,3, \ldots) .
\end{gathered}
$$

In a real physical system, there should be one root for (15). On the basis of an analysis in Section 4.1, where the sloshing

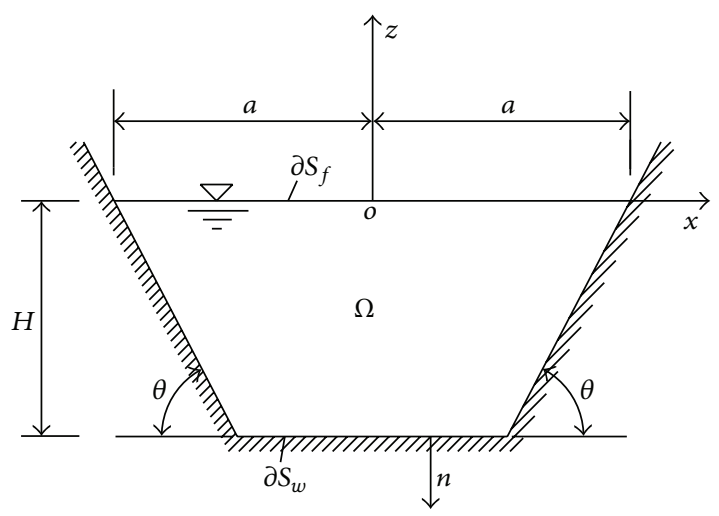

Figure 3: A trapezoid tank.
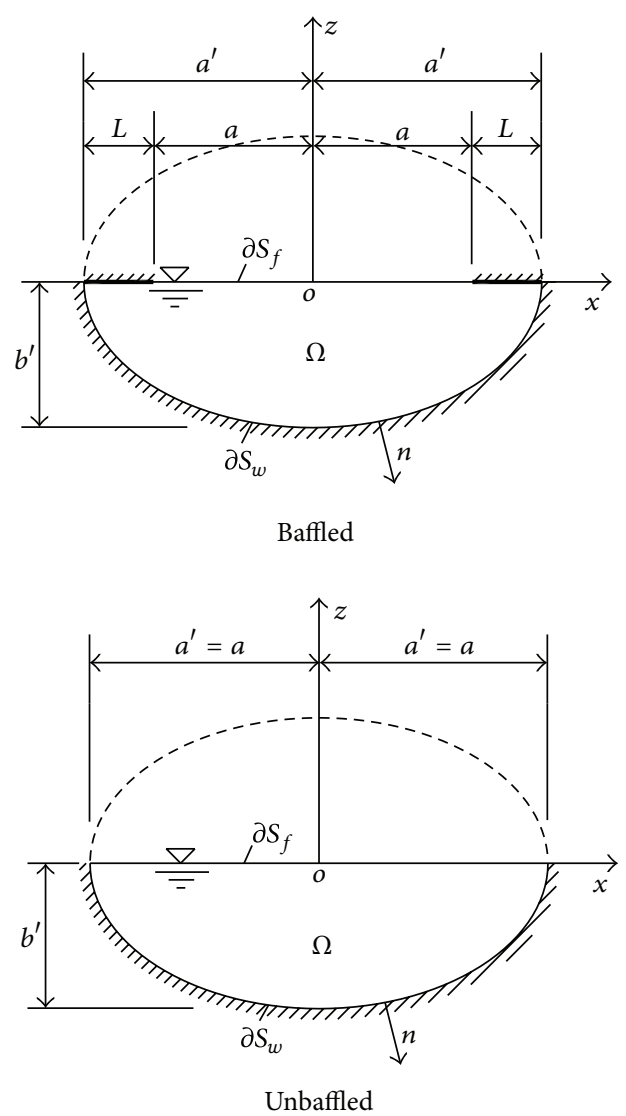

Figure 4: Baffled and unbaffled elliptical tanks.

frequencies of a rectangular tank are evaluated, the authentic roots of (15) can be determined as follows:

$$
\begin{gathered}
B_{j}=\frac{-b_{A j}-\sqrt{b_{A j}^{2}-4 \cdot a_{A j} \cdot c_{A j}}}{2 a_{A j}}, \quad(j=1,2,3, \ldots), \\
D_{j}=\frac{-b_{S j}-\sqrt{b_{S j}^{2}-4 \cdot a_{S j} \cdot c_{S j}}}{2 a_{S j}}, \quad(j=1,2,3, \ldots) .
\end{gathered}
$$




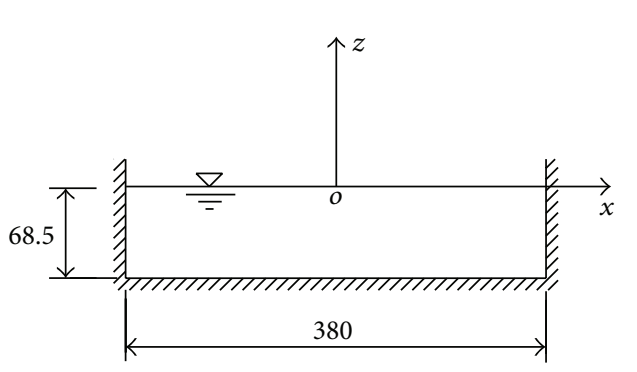

(a) Flat bottom

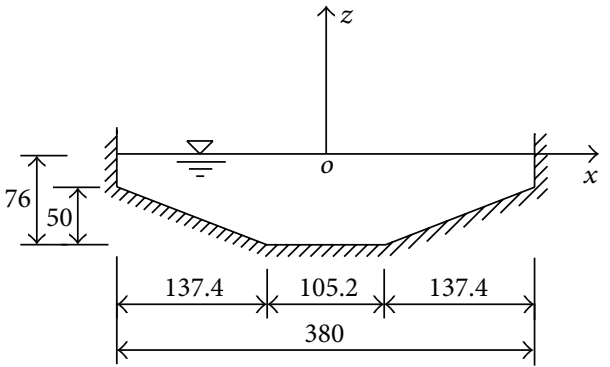

(b) V-shaped bottom

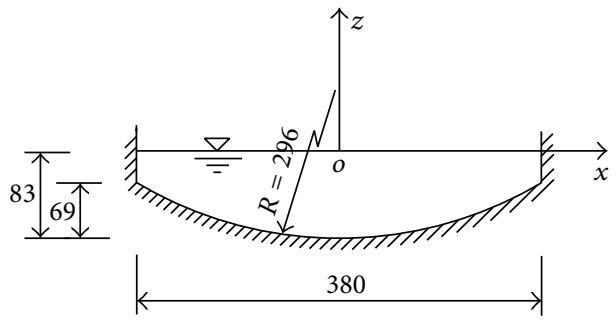

(c) Arc-shaped bottom

FIGURE 5: Various shape tuned liquid dampers (length unit: $\mathrm{mm}$ ).

The rest of the roots of (15) are the unwanted solutions, which are neglected here. For an arbitrary-section aqueduct, the coefficients $\left(a_{A j}, b_{A j}, c_{A j}\right)$ and $\left(a_{S j}, b_{S j}, c_{S j}\right)$ can be obtained by using (16) through integrals in the static liquid domain $\Omega$. Then, the coefficients $B_{j}$ and $D_{j}$ can be figured out by means of (17). Finally the antisymmetric and symmetric sloshing frequencies can be calculated by (14).

\section{Numerical Examples}

The analytical solution of this study is suitable for the fluid in an arbitrary-section aqueduct (tank). To validate this solution, the present method is applied to solve the sloshing frequencies of liquid in various shape tanks, and the frequency results of this study are compared with the other theoretical and experimental values.

4.1. Rectangular Tank. Figure 2 shows a rectangular tank, in which $2 a$ is the width of liquid and $H$ is the depth of liquid. On the basis of (13)-(17), the sloshing frequencies of liquid inside a rectangular tank can be derived as

$$
\omega_{j}^{2}=\left\{\begin{array}{c}
\frac{(2 j-1) \pi g}{2 a} \tanh \left[\frac{(2 j-1) \pi}{2 a} H\right] \\
\frac{j \pi g}{a} \tanh \left(\frac{j \pi}{a} H\right) \\
\text { antisymmetric }
\end{array} \quad(j=1,2,3, \ldots) .\right.
$$

It can be found that (18) is just the exact solution of sloshing frequencies. That is because the trigonometric functions in (12) are exactly the mode shape functions of the free fluid surface.

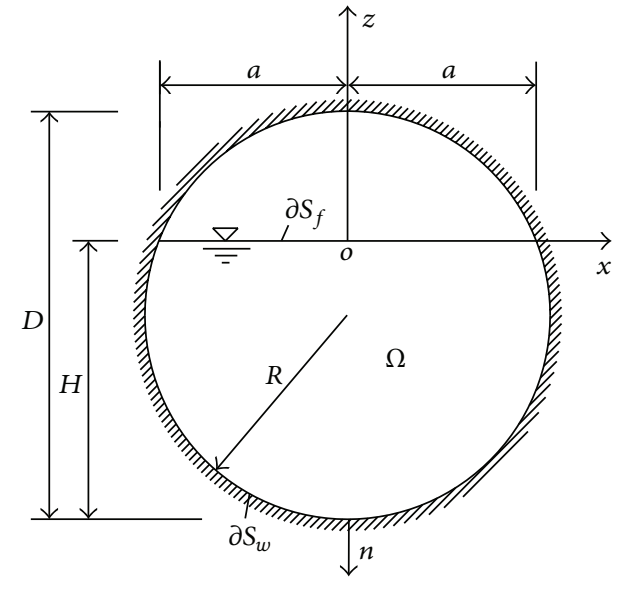

FIGURE 6: A circular-section aqueduct.

In this example, solving (15) can yield an extraneous root, which is apparently not the authentic solution of the liquid system and is discarded here.

4.2. Trapezoid Tank. Gardarsson [17] investigated the sloshing characteristics for a sloping bottom tank (isosceles trapezoid tank). In his PhD thesis, Gardarsson proposed a finite difference method for calculating the sloshing frequencies of fluid in a trapezoid tank. The tank configuration used for the experimental and theoretical analyses is sketched in Figure 3, in which the water depth $H$, half-width of the free surface $a$, and slope angle $\theta$ of side walls are fully presented in Table 1 .

The present approximate method is applied to calculate the fundamental frequencies of the three trapezoid tanks. 
TABLE 1: Comparison of the fundamental frequencies by Gardarsson [17] and present method.

\begin{tabular}{lccccc}
\hline$H(\mathrm{~m})$ & $a(\mathrm{~m})$ & $\theta($ degree $)$ & Gardarsson [17] theory $(\mathrm{Hz})$ & Gardarsson [17] experiment $(\mathrm{Hz})$ & Present method $(\mathrm{Hz})$ \\
\hline 0.04 & 0.1145 & $30^{\circ}$ & 1.2577 & 1.2812 & 1.2454 \\
0.07 & 0.166 & $30^{\circ}$ & 1.0875 & 1.0625 & 1.0889 \\
0.10 & 0.218 & $30^{\circ}$ & 0.9614 & 0.9375 & 0.9663 \\
\hline
\end{tabular}

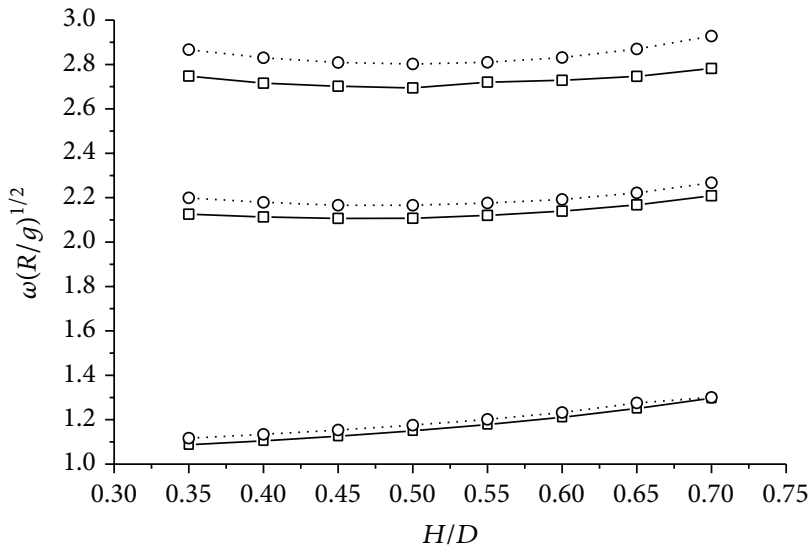

Antisymmetric frequencies - $\square$ - Finite element (ANSYS) ..o. Present study

(a) Antisymmetric frequency

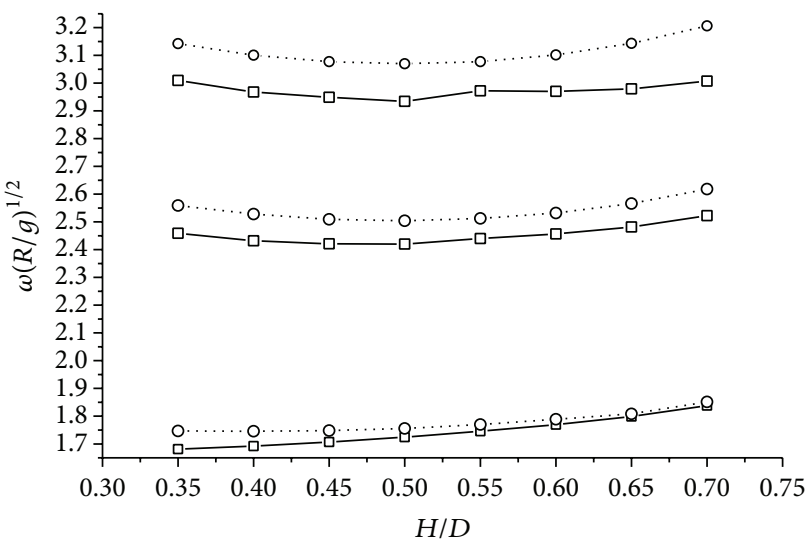

Symmetric frequencies - - Finite element (ANSYS) ..o. Present study

(b) Symmetric frequency

FIGURE 7: Variation $\omega(R / g)^{1 / 2}$ with liquid-depth ratio $H / D$ (circular aqueduct).

The results by Gardarsson and present approach are listed and compared in Table 1. It can be seen from Table 1 that the results of this study are within 3\% accuracy compared to the analytical and experimental values of Gardarsson. The solutions of this study have a good agreement with those by Gardarsson.

4.3. Semiellipse Tank. Hasheminejad and Aghabeigi [18] used a conformal transformation approach for studying the sloshing frequencies of fluid in half-full horizontal elliptical tanks. In the light of [18], the baffled and unbaffled ellipse container geometries are shown in Figure 4. A liquid fills the tanks to their half capacity, with the major and minor semiaxes and baffle length " $a$ ', " " $b$,", and " $L$," respectively. The symbol " $a$ " is the half width of still-liquid surface. For the un-baffled tank, the baffle length $L$ is zero (i.e., $a^{\prime}=a$ ).

One baffled and one unbaffled elliptic tanks from [18] are selected as the numerical examples. The dimensions of the tanks are indicated in Table 2. The first three normalized antisymmetric and symmetric sloshing frequencies $\left(\Omega_{j}=\right.$ $\left.\sqrt{a^{\prime} b^{\prime}} \omega_{j}^{2} / g(j=1,2,3)\right)$ by present study and [18] are displayed and compared in Table 2.

It can be observed from Table 2 that the maximum relative error of the normalized frequencies between [18] and present study is approximately $5 \%$; that is, the maximum relative error of the frequencies is around $2.5 \%$. The approximate results of this paper agree well with the analytical solutions of [18].

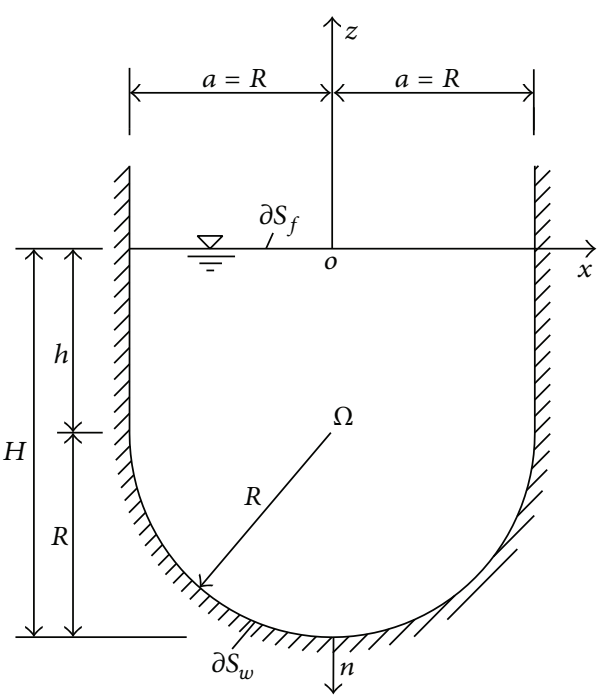

Figure 8: A U-shaped section aqueduct.

The solution of present paper not only applies to the semiellipse section but also to the ellipse tanks with arbitrary liquid depth.

4.4. Various Shape Tuned Liquid Dampers (TLD). Idir et al. [19] developed an equivalent bottom-wet-length method for calculating the fundamental frequencies of the various shape 
TABLE 2: The first three normalized antisymmetric and symmetric sloshing frequencies by [18] and present study $\Omega_{j}=\sqrt{a^{\prime} b^{\prime}} \omega_{j}^{2} / g,(j=$ $1,2,3)$.

\begin{tabular}{lccccccc}
\hline \multicolumn{2}{c}{ Unbaffled tank $\left(a^{\prime}=a=1.0 \mathrm{~m}, b^{\prime}=0.2 \mathrm{~m}\right)$} & \multicolumn{3}{c}{ Baffled tank $\left(a^{\prime}=1.0 \mathrm{~m}, b^{\prime}=0.2 \mathrm{~m}, L=0.05 \mathrm{~m}\right)$} \\
Antisymmetric & Symmetric & \multicolumn{3}{c}{$\begin{array}{c}\text { Antisymmetric } \\
\text { Symmetric }\end{array}$} \\
Present study & {$[18]$} & Present study & {$[18]$} & Present study & {$[18]$} & Present study & {$[18]$} \\
\hline$\Omega_{1} 0.19866$ & 0.19542 & 0.67487 & 0.66059 & 1.46170 & 1.43313 & 3.22321 & 3.21824 \\
$\Omega_{2} 1.34934$ & 1.29511 & 2.12996 & 2.05458 & 4.83482 & 4.91081 & 6.44640 \\
$\Omega_{3} 2.94933$ & 2.84342 & 3.76968 & 3.58803 & 8.05800 & 8.21077 & 9.66961 & 9.56741 \\
\hline
\end{tabular}

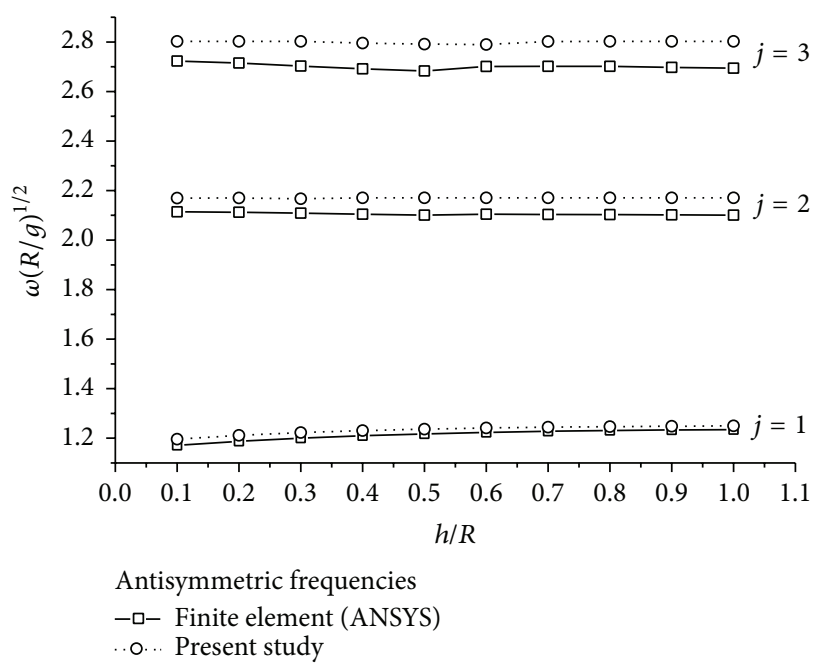

(a) Antisymmetric frequency

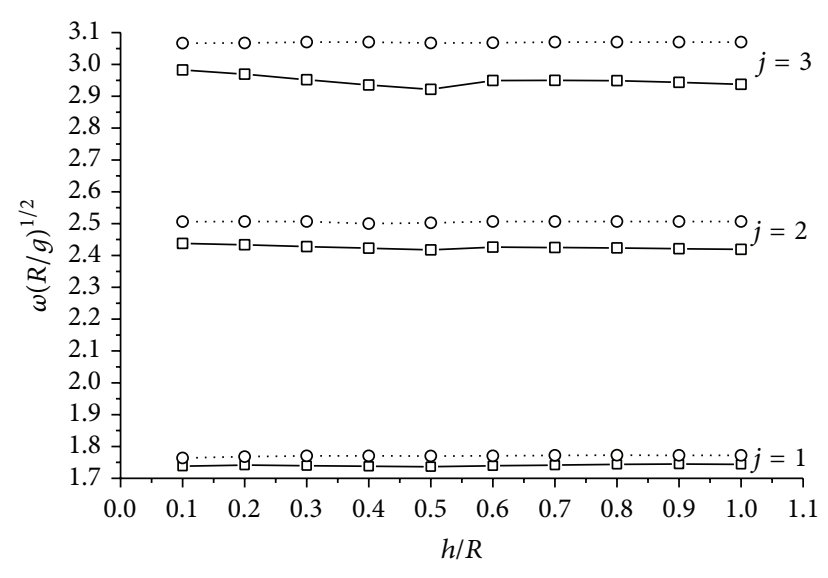

Symmetric frequencies - - Finite element (ANSYS) o. Present study

(b) Symmetric frequency

FIgURE 9: Variation of $\omega(R / g)^{1 / 2}$ with liquid-depth ratio $h / R$ (U-shaped aqueduct).

tuned liquid dampers (TLD). The three TLD geometries from [19], as shown in Figure 5, are presented here as the illustrative examples. The first antisymmetric (fundamental) sloshing frequencies by present approach and [19] are exhibited and compared in Table 3. Via comparison, the maximum relative error of the fundamental frequencies between [19] and present study is less than $3 \%$. The two results match well.

4.5. Circular Aqueduct. Figure 6 shows a circular-section aqueduct, in which $D$ is the inner diameter ( $R$ is the inner radius), $H$ is the liquid depth, and $a$ is the half width of the free liquid surface. Both the finite element and present approaches are used for computing the sloshing frequencies. The ANSYS Code [14] provides a fluid element (Fluid80) for simulating the sloshing problem of contained fluid. In this example, the parameters of fluid are chosen as mass density $\rho=1000\left(\mathrm{~kg} / \mathrm{m}^{3}\right)$, bulk modulus $E_{v}=2.067 \times 10^{9}(\mathrm{~Pa})$, and radius of aqueduct $R=3.0(\mathrm{~m})$. The viscosity coefficient of the fluid is assumed as zero (without damping). The interface between liquid and the rigid aqueduct is treated as a slip boundary condition, such that the normal relative displacement of the fluid and rigid container on the interface is forced to be zero and the tangential relative displacement on the interface is not restrained. Many obtained results using ANSYS Code have been verified by the previous investigations $[15,16]$. For example, in $[16]$ the fundamental
TABLE 3: Comparison of the fundamental frequencies by Idir et al. [19] and present study.

\begin{tabular}{lccc}
\hline Bottom shape & $\begin{array}{c}\text { Idir et al. [19] } \\
\text { theory (Hz) }\end{array}$ & $\begin{array}{c}\text { Idir et al. [19] } \\
\text { experiment } \\
(\mathrm{Hz})\end{array}$ & $\begin{array}{c}\text { Present study } \\
(\mathrm{Hz})\end{array}$ \\
\hline Flat & 1.0270 & 1.0050 & 1.0257 \\
V-shaped & 1.0270 & 1.0310 & 1.0279 \\
Arc-shaped & 1.0270 & 1.0550 & 1.0558 \\
\hline
\end{tabular}

frequencies in the rectangular tanks by ANSYS Code and the exact solution [3] were compared with each other, and the results showed the two solutions have a good agreement.

When the liquid depth $H$ varies from $0.35 D$ to $0.70 D$, the corresponding sloshing frequencies are both extracted by the finite element and present methods. Figures 7(a) and 7(b) show the variation of $\omega(R / g)^{1 / 2}$ with liquid-depth ratio $H / D$. It can be drawn from Figure 7 that the maximum relative error between present study and finite element is not greater than $5.0 \%$. The two results match well.

4.6. U-Shaped Aqueduct. Figure 8 shows a U-shaped aqueduct, in which $R$ is the inner radius of the bottom semicircle and $H(H=h+R)$ is the liquid depth. The computing methods and parameters of the fluid are the same as in Section 4.5. 
The liquid depth above the bottom semicircle is fixed as $h=$ $1.5 \mathrm{~m}$. When the liquid-depth ratio $h / R$ varies from 0.1 to 1.0 , the corresponding sloshing frequencies can be acquired by ANSYS Code and present method. Figures 9(a) and 9(b) show the variation of $\omega(R / g)^{1 / 2}$ with liquid-depth ratio $h / R$.

It can be observed from Figure 9 that the results of this study have a good consistency with those by ANSYS Code (finite element). The maximum error between present study and finite element is less than $5.0 \%$.

It can be noted from Figures 7 and 9 that the results of present study are slightly greater than those of finite element (ANSYS Code). This may be attributed to the compressibility of the fluid. A compressible fluid was applied in ANSYS Code, while an incompressible liquid was used in present study. The compressible case can produce lower natural frequencies. According to [16], the sloshing frequencies in a rectangular tank by ANSYS Code are slightly lower than the exact solutions using (18).

\section{Conclusion}

An approximate analytical solution of sloshing frequencies was formulated for a liquid in the arbitrary-section aqueducts. This approximate method has been, respectively, applied for finding the sloshing frequencies of the liquid in the various shape tanks (aqueducts). The first three antisymmetric and symmetric frequencies by this study in the various shape tanks are within $5 \%$ accuracy compared to the other analytical, numerical, and experimental values. The present approximate solutions for the various shape aqueducts are acceptable to the engineering applications. This method can be a simple and convenient way to estimate the sloshing frequencies of the liquid in various shape aqueducts for practical usage.

\section{Conflict of Interests}

The authors declare that there is no conflict of interests regarding the publication of this paper.

\section{Acknowledgment}

This study was supported by the National Science Foundation of China (Grant no. 51279133) which is gratefully acknowledged.

\section{References}

[1] H. Morand and R. Ohayon, Fluid Structure Interaction: Applied Numerical Methods, John Wiley \& Sons, New York, NY, USA, 1995.

[2] F. T. Dodge, The New "Dynamic Behavior of Liquids in Moving Containers", Southwest Research Institute, San Antonio, Tex, USA, 2000.

[3] R. A. Ibrahim, Liquid Sloshing Dynamics: Theory and Applications, Cambridge University Press, Cambridge, UK, 2005.

[4] O. M. Faltinsen and A. N. Timokha, Sloshing, Cambridge University Press, Cambridge, UK, 2009.
[5] H. R. Lawrence, C. J. Wang, and R. B. Reddy, "Variational solution of fuel sloshing modes," Jet Propulsion, vol. 28, no. 11, pp. 728-736, 1958.

[6] B. Budiansky, "Sloshing of liquids in circular canals and spherical tanks," Journal of Aerospace Sciences, vol. 27, no. 3, pp. 161173,1960

[7] D. W. Fox and J. R. Kuttler, "Sloshing frequencies," ZAMP Zeitschrift für Angewandte Mathematik und Physik, vol. 34, no. 5, pp. 668-696, 1983.

[8] P. McIver, "Sloshing frequencies for cylindrical and spherical containers filled to an arbitrary depth," Journal of Fluid Mechanics, vol. 201, pp. 243-257, 1989.

[9] D. V. Evans, "Wide-spacing approximation applied to multiple scattering and sloshing problems," Journal of Fluid Mechanics, vol. 210, pp. 647-658, 1990.

[10] D. V. Evans and C. M. Linton, "Sloshing frequencies," Quarterly Journal of Mechanics and Applied Mathematics, vol. 46, no. 1, pp. 71-87, 1993.

[11] R. A. Ibrahim, V. N. Pilipchuk, and T. Ikeda, "Recent advances in liquid sloshing dynamics," Applied Mechanics Reviews, vol. 54, no. 2, pp. 133-199, 2001.

[12] G. C. Everstine, "Structural analogies for scalar field problems," International Journal for Numerical Methods in Engineering, vol. 17, no. 3, pp. 471-476, 1981.

[13] H. C. Chen and R. L. Taylor, "Vibration analysis of fluid-solid systems using a finite element displacement formulation," International Journal for Numerical Methods in Engineering, vol. 29, no. 4, pp. 683-698, 1990.

[14] P. Kohnke, Ansys Theory Manual, ANSYS, 2001.

[15] G. C. Drosos, A. A. Dimas, and D. L. Karabalis, "Discrete models for seismic analysis of liquid storage tanks of arbitrary shape and fill height," Journal of Pressure Vessel Technology, vol. 130, no. 4, Article ID 041801, 12 pages, 2008.

[16] Y. Li, Q. Di, and Y. Gong, "Equivalent mechanical models of sloshing fluid in arbitrary-section aqueducts," Earthquake Engineering and Structural Dynamics, vol. 41, no. 6, pp. 1069-1087, 2012.

[17] S. Gardarsson, Shallow-Water Sloshing [Ph.D. thesis], University of Washington, Seattle, Wash, USA, 1997.

[18] S. M. Hasheminejad and M. Aghabeigi, "Liquid sloshing in halffull horizontal elliptical tanks," Journal of Sound and Vibration, vol. 324, no. 1-2, pp. 332-349, 2009.

[19] M. Idir, X. Ding, M. Lou, and G. Chen, "Fundamental frequency of water sloshing waves in a sloped-bottom tank as tuned liquid damper,' in Proceedings of the Structures Congress, pp. 1-10, Austin, Tex, USA, May 2009. 

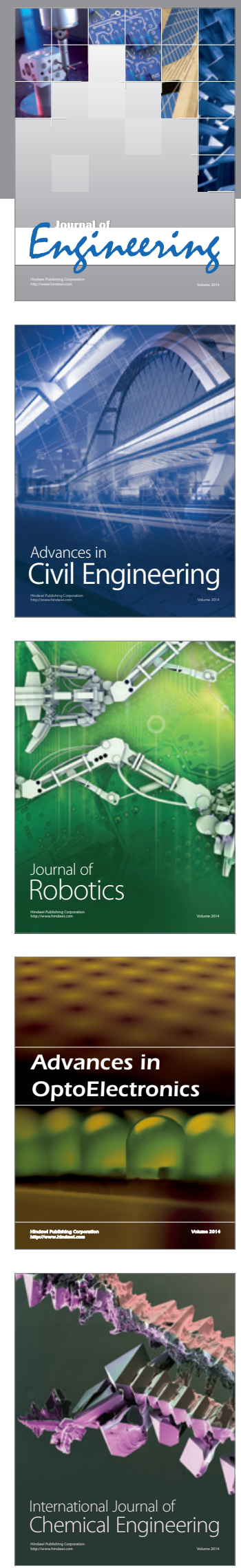

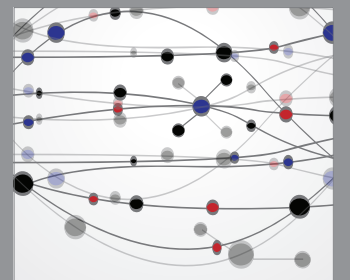

The Scientific World Journal
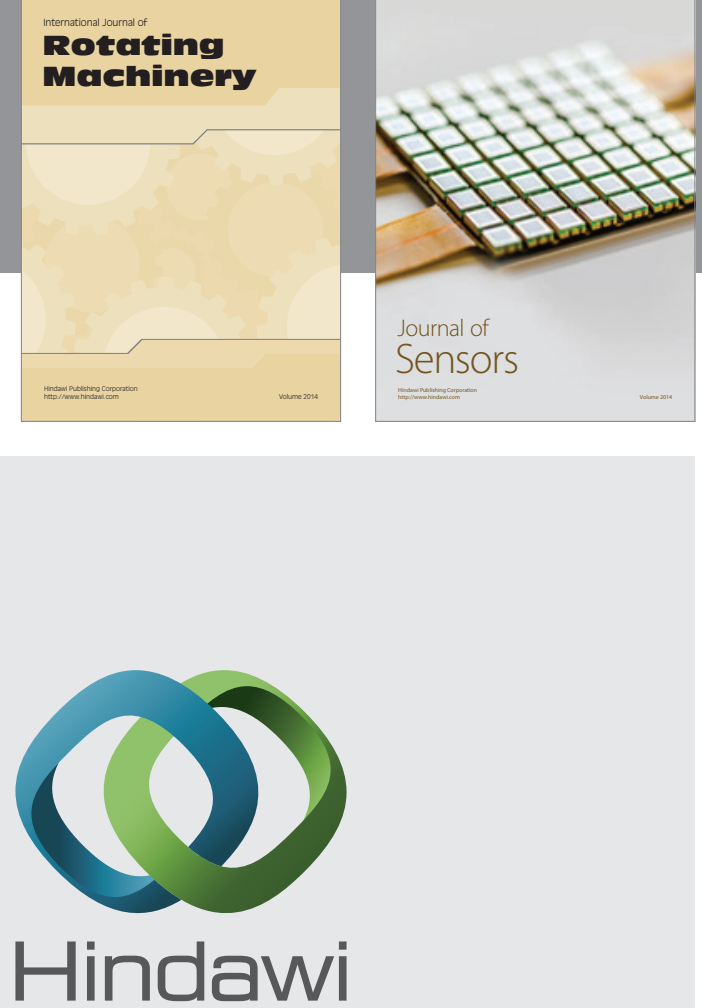

Submit your manuscripts at http://www.hindawi.com
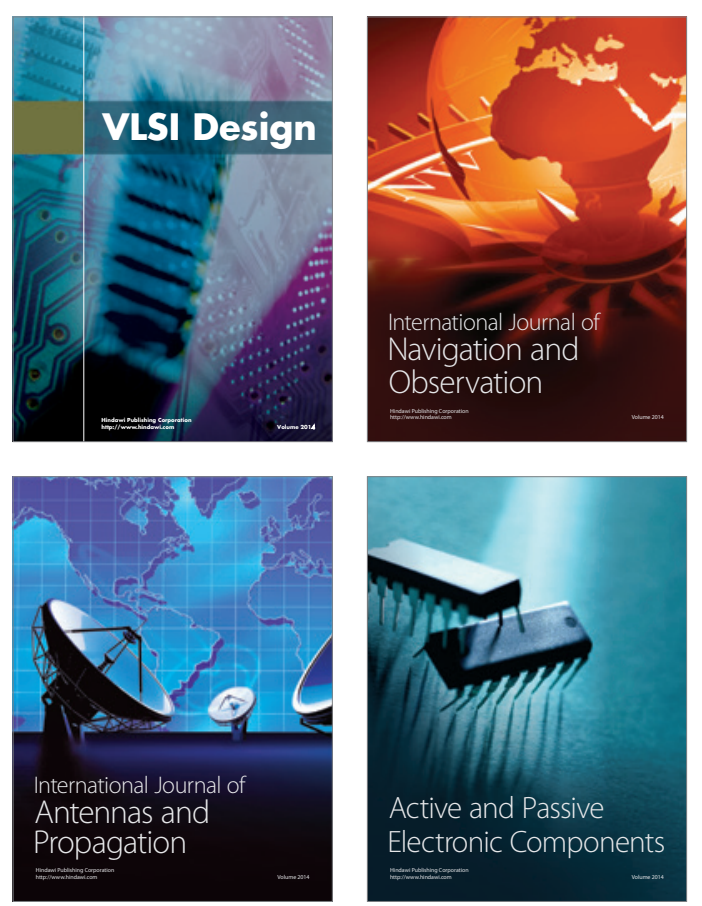
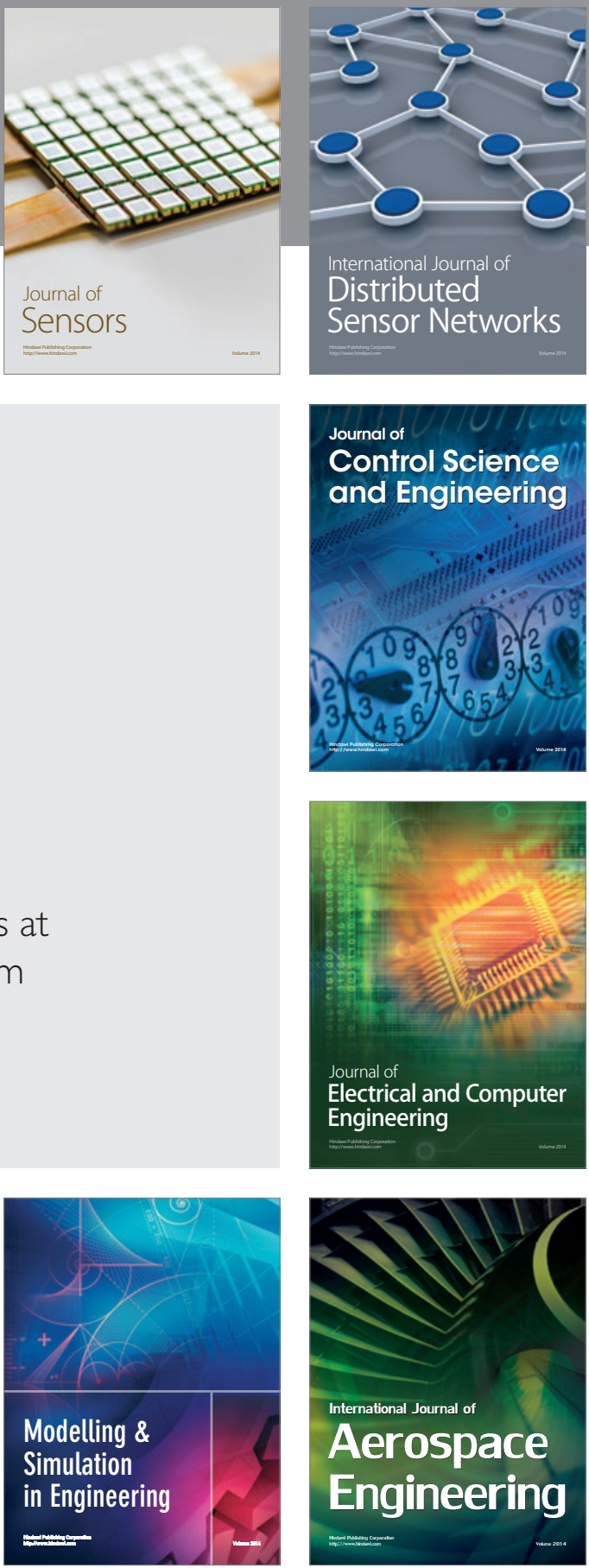

Journal of

Control Science

and Engineering
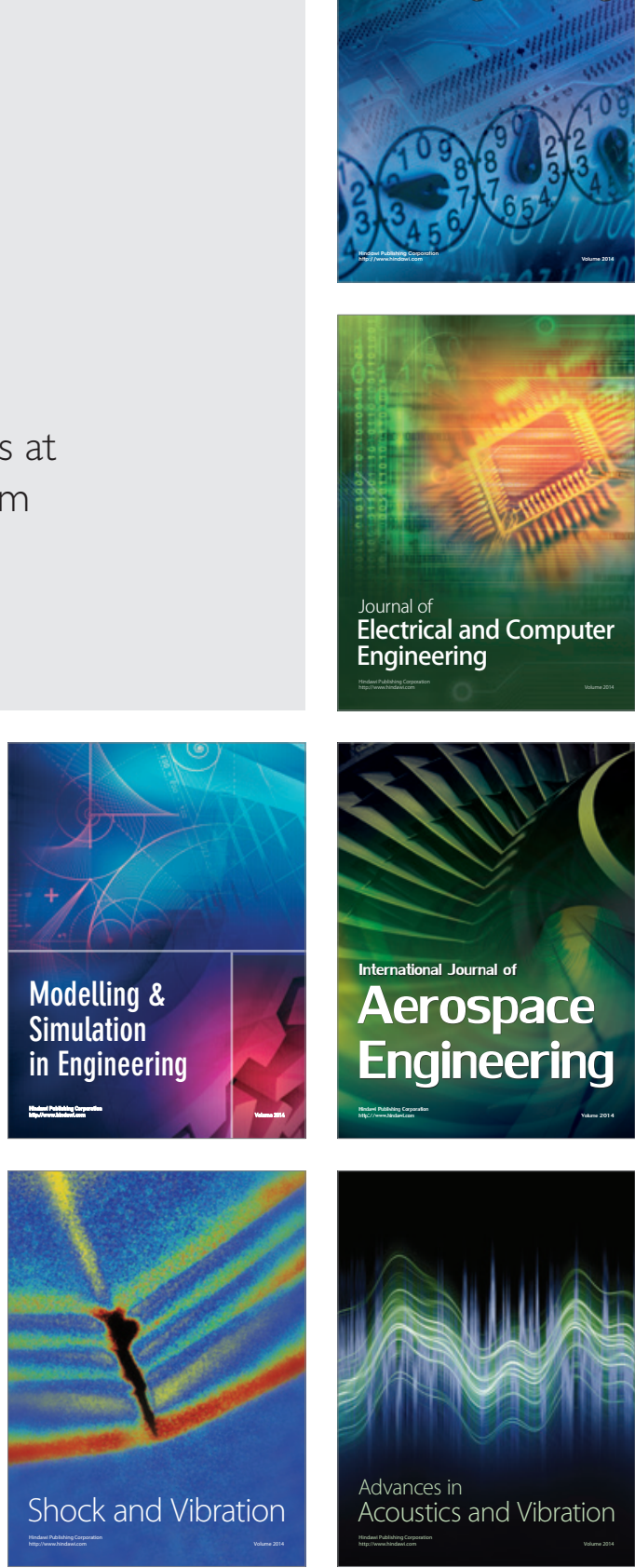